\title{
Rutin alleviates diabetic cardiomyopathy in a rat model of type 2 diabetes
}

\author{
YONG-BIN WANG ${ }^{1}$, ZHI-MING GE ${ }^{2}$, WEI-QIANG KANG ${ }^{3}$, \\ ZHE-XUN LIAN $^{4}$, JIAN YAO ${ }^{1}$ and CHANG-YONG ZHOU ${ }^{1}$ \\ ${ }^{1}$ Department of Emergency Medicine, The Affiliated Hospital of Qingdao University, Qingdao, Shandong 266003; \\ ${ }^{2}$ Department of Cardiology, Qilu Hospital of Shandong University, Jinan, Shandong 250014; \\ ${ }^{3}$ Qingdao Municipal Hospital, Qingdao, Shandong 266011; ${ }^{4}$ Department of Cardiology, \\ The Affiliated Hospital of Qingdao University, Qingdao, Shandong 266003, P.R. China
}

Received March 16, 2014; Accepted September 30, 2014

DOI: $10.3892 /$ etm.2014.2090

\begin{abstract}
Diabetic cardiomyopathy (DCM), an independent coronary heart disease that develops in diabetic individuals, is characterized by changes in the myocardial structure and function. The aim of the present study was to investigate the protective effect of rutin on DCM in a streptozotocin-induced diabetic rat model. Rutin was orally administrated at a dose of $8 \mathrm{mg} / \mathrm{kg}$ body weight. Metabolic profiles, myocardial enzymes and oxidative stress were examined by biochemical tests. The expression levels of cellular proteins associated with apoptosis were measured by western blot analysis, while the levels of inflammatory factors were assessed by immunohistochemical analyses. Rats with DCM exhibited an abnormal metabolic profile, aberrant myocardial enzymes, elevation of oxidative stress markers, increased levels of inflammatory factors and enhanced apoptotic cell death. Notably, rutin was shown to protect and improve myocardial dysfunction, oxidative stress, apoptosis and inflammation in the hearts of the diabetic rats. In conclusion, these results indicated that rutin may have great therapeutic potential in the treatment of DCM, and possibly other cardiovascular disorders, by preventing oxidative stress, inflammation and cell death. However, further detailed studies are required to reveal the exact mechanisms underlying the protective effect of rutin.
\end{abstract}

\section{Introduction}

Diabetes mellitus (DM) is accompanied by a number of complications due to the abnormal control of glycometabolism and lipid metabolism. Diabetic cardiomyopathy (DCM),

Correspondence to: Dr Yong-Bin Wang, Department of Emergency Medicine, The Affiliated Hospital of Qingdao University, 16 Jiangsu Road, Shinan, Qingdao, Shandong 266003, P.R. China

E-mail: wangyongbin369@gmail.com

Key words: diabetic cardiomyopathy, rutin, diabetic rats, streptozocin, antioxidants a condition observed in diabetic individuals, is characterized by changes to the myocardial structure and function, independent of coronary artery disease and systemic hypertension $(1,2)$. An increase in the levels of blood lipoproteins and free fatty acids facilitates the development of cardiovascular diseases, including hyperlipidemia and coronary artery disease, which can lead to further complications, such as retinopathy, nephropathy, neurosis, nephrotoxicity and hyperglycemia-induced coma (3). However, the development of DCM remains poorly understood and the underlying mechanisms have not yet been clearly elucidated. Diabetic complications are generally considered to be the result of oxidative stress (4), the excessive production of reactive oxygen species (ROS) and the aberration of the antioxidant system (5). In addition, diabetic complications are interrelated with the inflammatory response, and have been shown to be accelerated under a hyperglycemic state for the production of acute response factors in fat cells (6-8).

Rutin is a phenolic compound and flavonoid glycoside that is found in flowers and fruits as a major source. Rutin can be broadly extracted from nature sources, including buckwheat, oranges, grapes, lemons, limes, peaches and berries $(9,10)$. The compound has been reported to possess dynamic pharmacological functions, including antioxidant, antibactericidal, antiviral $(11,12)$, antitumor (13), anti-inflammatory (14), myocardial protection (15) and hepatoprotective (16) effects. In addition, previous studies have demonstrated the efficiency of the pharmacological functions of rutin as an antioxidant $(11,17,18)$.

In the present study, considering the potential therapeutic properties of rutin, the aim was to investigate the protective effects of rutin on DCM and its involvement in the alterations of cardiac function and associated mechanisms in a rat model of DM.

\section{Materials and methods}

Experimental animals. Two-month-old male Wistar rats were procured from the Chinese People's Liberation Army Military Academy of Medical Sciences Animal Experiment Center (Beijing, China). In total, 24 male Wistar rats (weight, 70-90 g) 
were used for the experiment. The animals were maintained with good ventilation and a 12-h light/dark cycle. Prior to the experiments, the animals were provided with food and water ad libitum. The animals were treated in accordance with the Guide for the Care and Use of Laboratory Animals published by the National Institutes of Health (NIH Publication no. 85-23, revised 1996). All experiments were approved by Institutional Animal Care and Use Committee of the Affiliated Hospital of Qingdao University (Qingdao, China).

$D M$ induction and rutin administration. To induce DM, the rats were fasted for $12 \mathrm{~h}$, after which $65 \mathrm{mg} / \mathrm{kg}$ streptozotocin (STZ) dissolved in 0.1 M citrate buffer $(\mathrm{pH} 4.5)$ was intraperitoneally administered. The rats were fasted again for $12 \mathrm{~h}$. At day 6 following STZ administration, the level of blood glucose was measured by collecting whole blood from the tail vein. Subsequently, the rats that had a blood glucose level of $>350 \mathrm{mg} /$ $\mathrm{dl}$ were screened for further experiments. The blood glucose level was measured using a glucometer (Accu-Chek Go model GS; Roche Diagnostics GmbH, Mannheim, Germany).

For the experiments, the rats were divided into three groups, which included the normal group (normal, $n=8$ ), STZ-induced $\mathrm{DM}$ group $(\mathrm{DM}, \mathrm{n}=8)$ and rutin-treated $\mathrm{DM}$ group $(\mathrm{DM}+$ rutin, $\mathrm{n}=8$ ). For the $\mathrm{DM}+$ rutin group, $8 \mathrm{mg} / \mathrm{kg}$ rutin dissolved in soybean oil was orally administered at the same time every day for one week following the induction of DM.

Hematological analysis. At $72 \mathrm{~h}$ following the STZ injection, blood glucose levels were measured using a glucometer (Changsha Sinocare Inc., Changsha, China), following tail vein puncture blood sampling. Serum triglyceride (TG) and total cholesterol (TC) levels were determined using an auto-biochemical analysis system (AU2700; Olympus, Tokyo, Japan). The body weight was recorded every day for one week. After 12 days of rutin treatment, the experimental animals were euthanized by $\mathrm{CO}_{2}$ inhalation.

Measurement of serum myocardial enzymes. Blood samples were collected from the abdominal artery and the serum was separated by centrifugation at $1,600 \mathrm{x}$ for $10 \mathrm{~min}$ at $4^{\circ} \mathrm{C}$. The levels of creatine kinase-MB (CK-MB), lactate dehydrogenase (LDH) and aspartate aminotransferase (AST) were determined using an auto-biochemical analysis system (AU2700; Olympus).

Estimation of the superoxide dismutase (SOD) activity and malondialdehyde (MDA) level. Heart tissue samples were weighed and homogenized (1:10, w/v) in $50 \mathrm{mmol} / \mathrm{l}$ phosphate buffer ( $\mathrm{pH}$ 7.4). The SOD activity and MDA level were measured using the appropriate detection kits A001-4 for SOD and A003-1 for MDA purchased from Nanjing Jiancheng Bioengineering Institute (Nanjing, China).

Immunohistochemical staining. Paraffin-embedded sections underwent immunohistochemistry using a microwave-based antigen retrieval method. The sections were incubated with primary rabbit polyclonal anti-tumor necrosis factor- $\alpha$ (TNF- $\alpha$; Abcam, Cambridge, MA, USA; \#ab9635; dilution: $1 \mu \mathrm{g} / \mathrm{ml}$ ) and anti-interleukin-6 (IL-6; Abcam; \#ab6672; 1:500) antibodies overnight, and subsequently with a corresponding biotinylated anti-rabbit (\#7074) and anti-mouse $\operatorname{IgG}$ (\#7076) secondary antibody (Cell Signaling Technology, Inc., Danvers, MA, USA) for $30 \mathrm{~min}$ at $37^{\circ} \mathrm{C}$. Negative controls were performed with the omission of the primary antibody. The results were viewed under a confocal FV1000 SPD laser-scanning microscope (Olympus).

Western blot analysis. Frozen left ventricular tissue samples were homogenized in ice-cold lysis buffer [20 mM Tris (pH 7.5), 150 mM NaCl, 1 mM EDTA, 1 mM EGTA, 1\% Triton X-100, $2.5 \mathrm{mM}$ sodium pyrophosphate, $1 \mathrm{mM}$ $\beta$-glycerolphosphate, $1 \mathrm{mM} \mathrm{Na} \mathrm{VO}_{4}, 1 \mathrm{mg} / \mathrm{ml}$ aprotinin leupeptin and pepstatin and $1 \mathrm{mM}$ phenylmethylsulfonyl fluoride] and centrifuged at $1,600 \mathrm{x}$ g for $15 \mathrm{~min}$ at $4^{\circ} \mathrm{C}$. A bicinchoninic acid (BCA) protein assay (Beyotime Institute of Biotechnology, Haimen, China) was utilized to measure the protein concentration in the supernatant. Equal amounts of protein were used for western blot analysis, which was performed with the following antibodies: Caspase-3 (\#9661; 1:1000), Bcl-2 (\#2870; 1:1,000) and BAX (\#2772; 1:1000; all from Cell Signaling Technology, Inc.), and $\beta$-actin (\#sc47778; 1:1000; Santa Cruz Biotechnology, Inc., Dallas, TX, USA). The membrane was incubated with a horseradish peroxidase-conjugated secondary antibody for $1 \mathrm{~h}$ at $37^{\circ} \mathrm{C}$. Blots were developed using an enhanced chemiluminescence kit (Pierce Biotechnology, Inc. Rockford, IL, USA).

Statistical analysis. Data are presented as the mean \pm standard error of the mean. SPSS software version 22 (SPSS, Inc., Chicago, IL, USA) was used for the statistical analysis to perform one-way analysis of variance, where $\mathrm{P}<0.05$ was considered to indicate a statistically significant difference.

\section{Results}

Rutin prevents metabolic abnormalities. Hematological analysis revealed the metabolic characteristics of the experimental animals (Table I). In the DM group, STZ-induced diabetic rats exhibited a markedly lower body weight and higher blood glucose levels when compared with the control group $(\mathrm{P}<0.05)$. In addition, the heart-to-body weight ratio $(\mathrm{HW} / \mathrm{BW})$ in the DM group was significantly $(\mathrm{P}<0.05)$ higher compared with the control and rutin-treated groups, respectively (Table 1). Furthermore, rutin was shown to significantly decrease the blood glucose levels (to similar values to the control group) in the diabetic rats $(\mathrm{P}<0.05)$. In the basal fasting state, the DM group exhibited significantly $(\mathrm{P}<0.05)$ higher levels of TG and TC when compared with the control group. However, the level of TG was decreased in the $\mathrm{DM}+$ rutin group compared to the DM group. No statistically significant difference in the TC level was observed between the DM and DM + rutin groups.

Rutin inhibits myocardial injury and oxidative stress. The myocardial enzymes, CK-MB, LDH and AST, can be used as biochemical indicators of myocardial injury (Fig. 1A). When compared with the control group, the levels of the three enzymes were significantly increased in the DM group $(\mathrm{P}<0.05)$. In the rutin-treated DM group, decreased levels of myocardial enzymes were observed ( $\mathrm{P}<0.05$, vs. DM group); thus, rutin was shown to protect the diabetic rats against cardiac injury. 
Table I. Rutin prevents metabolic abnormalities.

\begin{tabular}{|c|c|c|c|c|c|}
\hline Group & Body weight (g) & HW/BW (mg/g) & Blood glucose (mmol/l) & TG $(\mathrm{mmol} / \mathrm{l})$ & $\mathrm{TC}(\mathrm{mmol} / \mathrm{l})$ \\
\hline Control & $415 \pm 16$ & $2.77 \pm 0.16$ & $5.3 \pm 0.3$ & $0.79 \pm 0.07$ & $1.26 \pm 0.07$ \\
\hline $\mathrm{DM}$ & $257 \pm 17^{\mathrm{a}}$ & $4.25 \pm 0.18^{\mathrm{a}}$ & $21.5 \pm 1.2^{\mathrm{a}}$ & $1.24 \pm 0.11^{\mathrm{a}}$ & $1.51 \pm 0.09^{\mathrm{a}}$ \\
\hline $\mathrm{DM}$ + rutin & $345 \pm 21$ & $3.32 \pm 0.19$ & $9.2 \pm 1.9^{b}$ & $0.93 \pm 0.04$ & $1.35 \pm 0.12$ \\
\hline
\end{tabular}

Body weight and heart weight were measured on the day that the rats were sacrificed. Blood glucose, TG and TC levels were measured in the basal fasting state on the day that the rats were sacrificed. Data are expressed as the mean \pm standard error of the mean; ${ }^{\text {a }}<0.05$, vs. control group; ${ }^{\mathrm{b}} \mathrm{P}<0.05$, vs. DM group ( $\mathrm{n}=8$ per group). TG, triglycerides; $\mathrm{TC}$, total cholesterol; HW/BW, heart-to-body weight ratio; DM, diabetes mellitus.
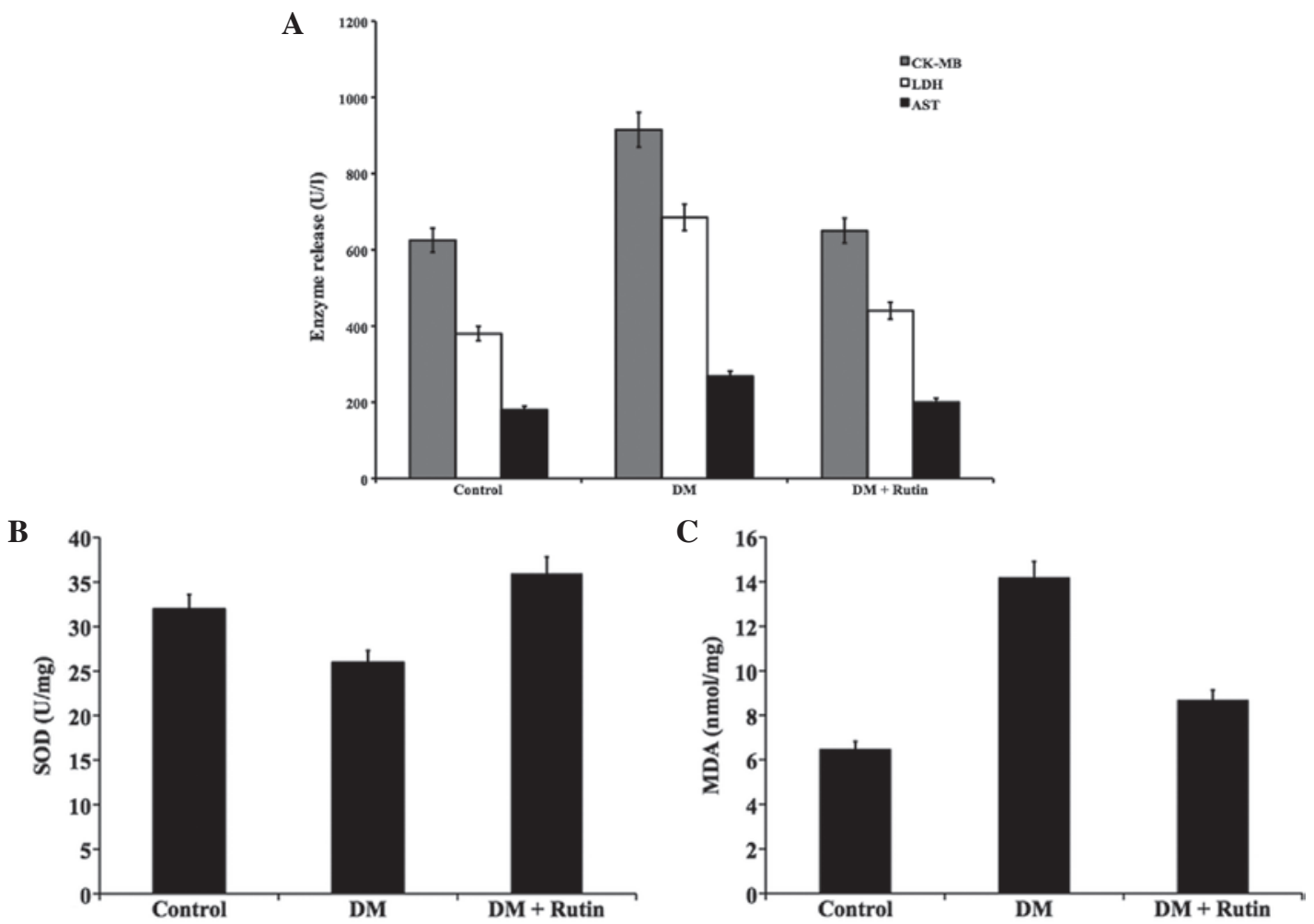

Figure 1. Rutin prevents myocardial injury and oxidative stress in an experimental model of DM in rats. Rutin was shown to (A) inhibit serum myocardial enzyme release, (B) increase SOD activity and (C) decrease the MDA content in the heart tissue. MDA, malondialdehyde; CK-MB, creatine kinase-MB; LDH, lactate dehydrogenase; AST, aspartate aminotransferase; SOD, superoxide dismutase; DM, diabetes mellitus.

In the heart tissue samples of the DM group rats, a decrease in the activity of SOD (Fig. 1B) and an increase in the accumulation of lipid peroxides with a concordant increase in MDA content (Fig. 1C) were observed ( $\mathrm{P}<0.05$, vs. control group). Following treatment with rutin in the diabetic rats, the activity of SOD was found to be upregulated, while the MDA content was markedly decreased ( $\mathrm{P}<0.05$, vs. DM group).

Rutin prevents the production of inflammatory factors. Immunohistochemical analysis revealed increased staining for the inflammatory factors, TNF- $\alpha$ and IL-6, in the DM group when compared with the control group. However, decreased levels of staining (TNF- $\alpha$ and IL-6) were observed in the rutin-treated group when compared with the DM group (Fig. 2). These observations indicate the protective effect of rutin against inflammation.
Rutin inhibits DM-induced apoptosis of cardiomyocytes. The expression levels of the antiapoptotic protein, Bcl-2, and proapoptotic proteins, BAX and caspase-3, were assessed by immunoblotting. The blots revealed enhanced expression levels of caspase- 3 and BAX, but a reduced expression of Bcl- 2 in the DM group when compared with the control group. Notably, the diabetic rats treated with rutin exhibited significantly increased protein expression levels of $\mathrm{Bcl}-2$, and downregulated protein expression levels of caspase-3 and BAX (Fig. 3).

\section{Discussion}

The metabolic abnormalities observed in the DM group, including the markedly higher concentrations of plasma and serum glucose $(\mathrm{P}<0.05)$, were the result of insulin secretion inhibition caused by the ROS produced by STZ. The ROS 

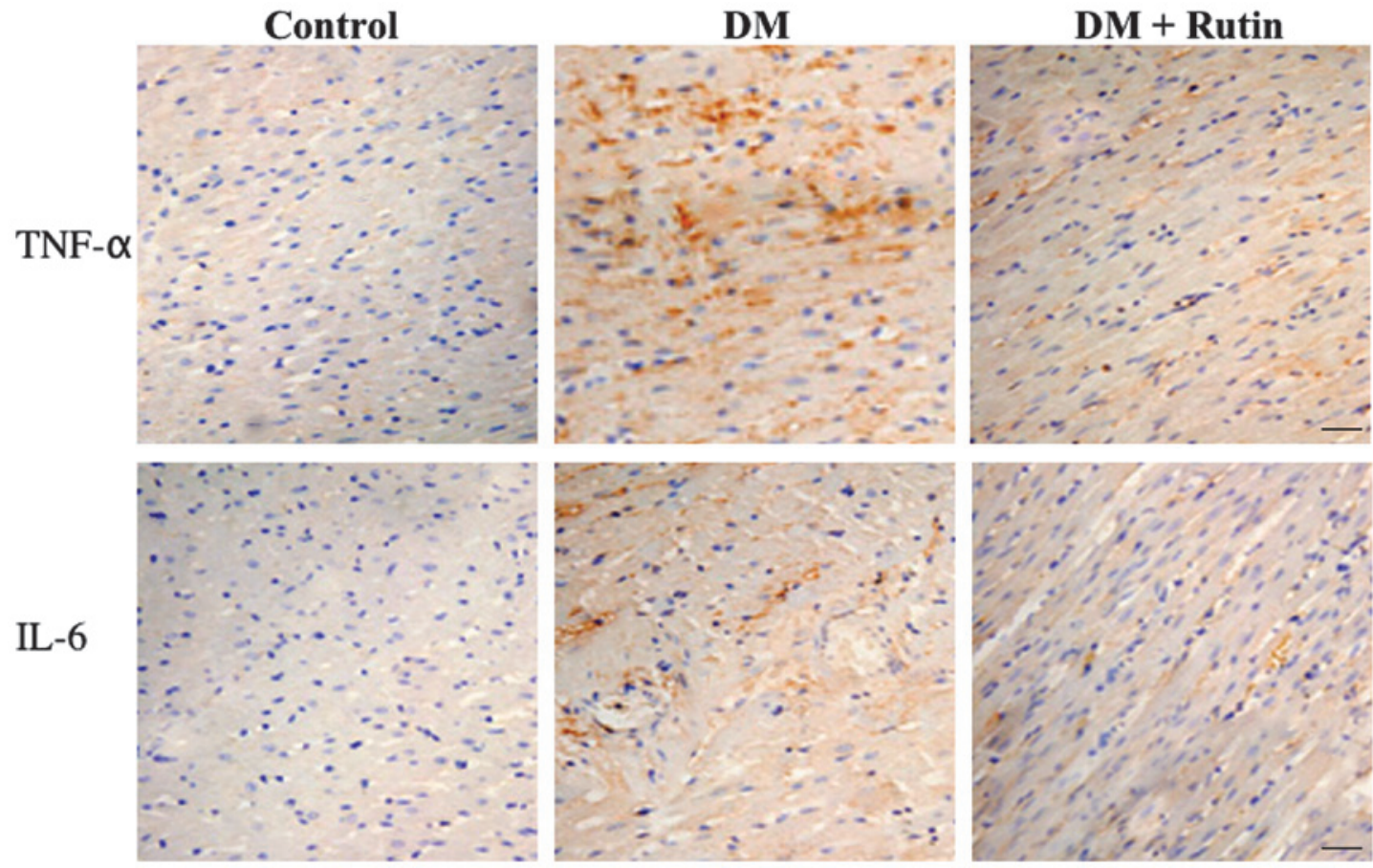

Figure 2. Immunohistochemical staining for myocardial TNF- $\alpha$ and IL-1 $\beta$ expression. Brown staining indicates the cells with positive expression (scale bar, $50 \mathrm{~mm}$; magnification, $\mathrm{x} 20$ ). DM, diabetes mellitus; TNF, tumor necrosis factor; IL, interleukin.

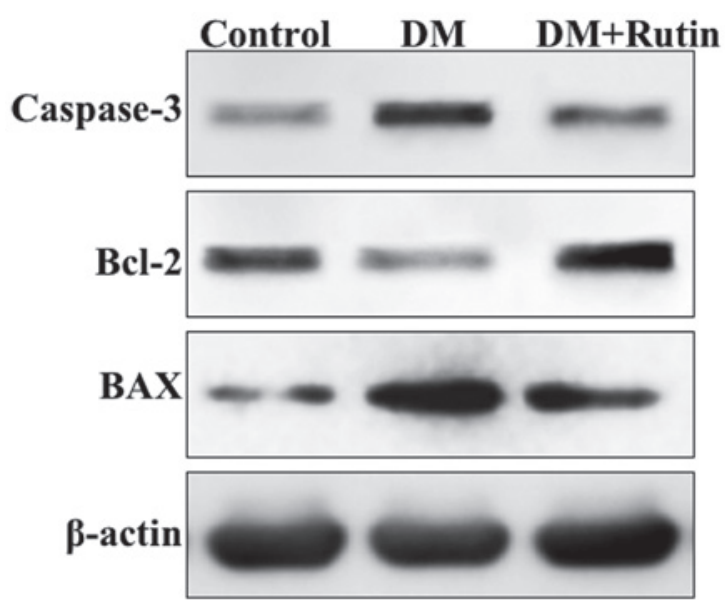

Figure 3. Western blot analysis showing the protein expression levels of caspase-3, Bcl-2 and BAX. $\beta$-actin was used as a control. DM, diabetes mellitus.

subsequently repressed the function of the antioxidant system, while causing oxidative damage to the pancreatic $\beta$-cells (19). Although the concentration levels of plasma and serum glucose in the DM + rutin group were not reduced to the same extent as that observed in the control group, the levels were significantly decreased compared with the level in the DM group $(\mathrm{P}<0.05)$. As shown in the study by Kamalakkannan and Prince (20), the concentration levels of plasma glucose and serum glucose decreased as rutin removed free radicals and repressed lipid peroxidation, while protecting the $\beta$-cells by impeding the oxidative stress caused by STZ and increasing the level of insulin secretion. The different forms of DM include type 1 (insulin-dependent), type 2 (non-insulin-dependent) and gestational diabetes. Using a low dose of STZ combined with a high-energy intake is considered to be a general strategy to obtain an animal model of type $2 \mathrm{DM}$, since these factors simulate the real course of the human disease $(21,22)$. In the present study, STZ injections were shown to be successful in inducing DM by markedly elevating the levels of serum glucose, TG and TC. Through using this method, the serum symptoms exhibited an increased similarity to those of type 2 DM compared with those of type 1 DM.

DCM is classified as ventricular dysfunction with an increased risk of cardiac failure, in the absence of hypertension, coronary artery and valvular heart diseases (23). The condition is frequently observed in humans and animals. Consistent with a previous study (24), the untreated DM rats in the present study were characterized by a decreased or attenuated antioxidant defense, as shown by the decreased SOD activity, accompanied with increased myocardial lipid peroxidation and inactivation of prosurvival pathways of Bcl-2, eventually culminating in cell apoptosis and increased levels of inflammation. By contrast, the administration of rutin was demonstrated to prevent the development of these characteristic alterations of DCM. The beneficial effects of rutin may be explained in part as follows. Firstly, rutin treatment was shown to decrease the elevated levels of blood glucose and TG. A previous study demonstrated that the onset of cardiovascular complications may be delayed by controlling metabolic abnormalities (25).

Secondly, rutin was found to attenuate oxidative stress. The compound has previously been demonstrated to intercept and neutralize ROS using its potential antioxidant function (17). Oxidative stress is defined as an imbalance between the production and elimination of free radicals, which play a critical role in the development of heart failure and left ventricular remodeling in DCM (26). Hyperglycemia has been shown to exacerbate glucose oxidation and the generation of ROS in 
the mitochondria (27), which subsequently results in DNA damage and an accelerated rate of apoptosis. NADPH oxidase is a critical determinant of myocardial ROS generation (28). In the present study, rutin was demonstrated to decrease the level of SOD activity and reduce lipid peroxidation in a rat model of DM.

Thirdly, rutin was shown to suppress cardiac inflammation, which is characterized by increased levels of proinflammatory cytokines. Proinflammatory cytokines, including IL-6 and TNF- $\alpha$, are critical in the manifestation of DCM (29). Rutin has a number of properties, including antioxidant activities, anti-inflammatory effects (14), myocardial protection (15) and hepatoprotective activities (16), that enable the suppression of cardiac inflammation. Rutin is hypothesized to exert protective effects for various organs in DCM rats through dynamic medical functions. In addition, according to a previous study, antioxidants impede inflammation (30), and it is known that the antioxidative activity and anti-inflammatory effects of rutin are not an independent function.

In conclusion, the results demonstrated that rutin may have great therapeutic potential in the treatment of DCM, and possibly other cardiovascular disorders, by ameliorating metabolic abnormalities, oxidative stress, inflammation and cellular apoptosis pathways.

\section{References}

1. Acar E, Ural D, Bildirici U, Sahin T and Yilmaz I: Diabetic cardiomyopathy. Anadolu Kardiyol Derg 11: 732-737, 2011.

2. Battiprolu PK, Gillette TG, Wang ZV, Lavandero S and Hill JA: Diabetic cardiomyopathy: Mechanisms and therapeutic targets. Drug Discov Today Dis Mech 7: e135-e143, 2010.

3. West KM, Ahuja MM, Bennett PH, et al: The role of circulating glucose and triglyceride concentrations and their interactions with other 'risk factors' as determinants of arterial disease in nine diabetic population samples from the WHO multinational study. Diabetes Care 6: 361-369, 1983

4. Baynes JW: Role of oxidative stress in development of complications in diabetes. Diabetes 40: 405-412, 1991.

5. Coppey LJ, Gellett JS, Davidson EP, et al: Effect of antioxidant treatment of streptozotocin-induced diabetic rats on endoneurial blood flow, motor nerve conduction velocity, and vascular reactivity of epineurial arterioles of the sciatic nerve. Diabetes 50 1927-1937, 2001

6. Guha M, Bai W, Nadler JL and Natarajan R: Molecular mechanisms of tumor necrosis factor alpha gene expression in monocytic cells via hyperglycemia-induced oxidant stress-dependent and -independent pathways. J Biol Chem 275: 17728-17739, 2000.

7. Lin Y, Rajala MW, Berger JP, et al: Hyperglycemia-induced production of acute phase reactants in adipose tissue. J Biol Chem 276: 42077-42083, 2001.

8. Aronson D, Bartha P, Zinder O, et al: Obesity is the major determinant of elevated $\mathrm{C}$-reactive protein in subjects with the metabolic syndrome. Int J Obes Relat Metab Disord 28: 674-679, 2004.

9. Kreft S, Knapp M and Kreft I: Extraction of rutin from buckwheat (Fagopyrum esculentum Moench) seeds and determination by capillary electrophoresis. J Agric Food Chem 47: 4649-4652, 1999.
10. Huang WY, Zhang HC, Liu WX and Li CY: Survey of antioxidant capacity and phenolic composition of blueberry, blackberry, and strawberry in Nanjing. J Zhejiang Univ Sci B 13: 94-102, 2012.

11. Potter JD: Cancer prevention: epidemiology and experiment. Cancer Lett 114: 7-9, 1997.

12. Rice-Evans C and Packer L (eds): Flavonoids in Health and Diseases. Vol 13. Marcel Decker, New York, NY, pp483-522, 1998.

13. Deschner EE, Ruperto J, Wong G and Newmark HL: Quercetin and rutin as inhibitors of azoxymethanol-induced colonic neoplasia. Carcinogenesis 12: 1193-1196, 1991.

14. Aleksandrov PN, Speranskaia TV, Bobkov IuG, Zagorevskiǔ VA and Zykov DA: Effect of rutin and esculamine on models of aseptic inflammation. Farmakol Toksikol 49: 84-86, 1986 (In Russian).

15. Pozin VM, Skuratovskaia SG and Pocheptsova GA: Changes in the vascular wall and ischemic damages to the myocardium in reversible episodes of heart muscle ischemia. Fiziol Zh 42: 10-16, 1996 (In Russian).

16. Janbaz KH, Saeed SA and Gilani AH: Protective effect of rutin on paracetamol- and $\mathrm{CCl}_{4}$-induced hepatotoxicity in rodents. Fitoterapia 73: 557-563, 2002.

17. Nagasawa T, Tabata N, Ito Y, et al: Dietary G-rutin suppresses glycation in tissue proteins of streptozotocin-induced diabetic rats. Mol Cell Biochem 252: 141-147, 2003.

18. Nagasawa T, Tabata N, Ito Y, et al: Inhibition of glycation reaction in tissue protein incubations by water soluble rutin derivative. Mol Cell Biochem 249: 3-10, 2003.

19. Szkudelski T: The mechanism of alloxan and streptozotocin action in B cells of the rat pancreas. Physiol Res 50: 537-546, 2001.

20. Kamalakkannan N and Prince PS: Antihyperglycaemic and antioxidant effect of rutin, a polyphenolic flavonoid, in streptozotocininduced diabetic wistar rats. Basic Clin Pharmacol Toxicol 98: 97-103, 2006.

21. Wang HJ, Jin YX, Shen W, et al: Low dose streptozotocin (STZ) combined with high energy intake can effectively induce type 2 diabetes through altering the related gene expression. Asia Pac J Clin Nutr 16 (Suppl 1): 412-417, 2007.

22. Ti Y, Xie GL, Wang ZH, et al: TRB3 gene silencing alleviates diabetic cardiomyopathy in a type 2 diabetic rat model. Diabetes 60: 2963-2974, 2011.

23. Tarquini R, Lazzeri C, Pala L, Rotella CM and Gensini GF: The diabetic cardiomyopathy. Acta Diabetol 48: 173-181, 2011.

24. Yu W, Wu J, Cai F, et al: Curcumin alleviates diabetic cardiomyopathy in experimental diabetic rats. PLosOne 7: e52013, 2012

25. Boudina S and Abel ED: Diabetic cardiomyopathy revisited. Circulation 115: 3213-3223, 2007.

26. Rajesh M, Mukhopadhyay P, Bátkai S, et al: Cannabidiol attenuates cardiac dysfunction, oxidative stress, fibrosis, and inflammatory and cell death signaling pathways in diabetic cardiomyopathy. J Am Coll Cardiol 56: 2115-2125, 2010.

27. Wang J, Wang H, Hao P, et al: Inhibition of aldehyde dehydrogenase 2 by oxidative stress is associated with cardiac dysfunction in diabetic rats. Mol Med 17: 172-179, 2011.

28. Frantz S, Brandes RP, Hu K, et al: Left ventricular remodeling after myocardial infarction in mice with targeted deletion of the NADPH oxidase subunit gp91PHOX. Basic Res Cardiol 101: 127-132, 2006

29. Mano Y, Anzai T, Kaneko H, et al: Overexpression of human C-reactive protein exacerbates left ventricular remodeling in diabetic cardiomyopathy. Circ J 75: 1717-1727, 2011.

30. Cuzzocrea S, Riley DP, Caputi AP and Salvemini D: Antioxidant therapy: a new pharmacological approach in shock, inflammation, and ischemia/reperfusion injury. Pharmacol Rev 53: $135-159,2011$. 